\title{
AN INDIRECT SEARCH FOR WEAKLY INTERACTING MASSIVE PARTICLES IN THE SUN USING 3109.6 DAYS OF UPWARD-GOING MUONS IN SUPER-KAMIOKANDE
}

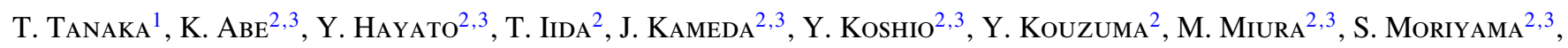

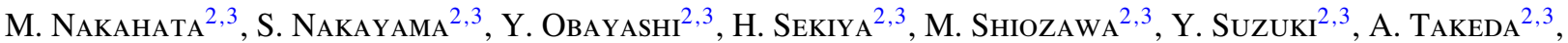

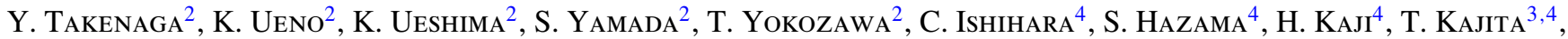
K. Kaneyuki ${ }^{3,4,33}$, T. McLachlan ${ }^{4}$, K. Okumura ${ }^{4}$, Y. Shimizu ${ }^{4}$, N. Tanimoto ${ }^{4}$, F. Dufour ${ }^{5}$, E. Kearns ${ }^{3,5}$, M. Litos $^{5}$,

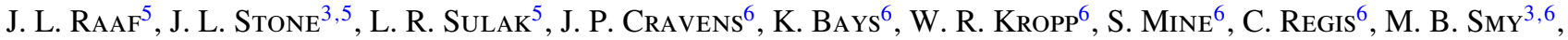

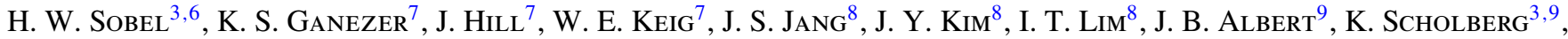
C. W. Walter ${ }^{3,9}$, R. Wendell ${ }^{9}$, T. WongJirad ${ }^{9}$, T. Ishizuka ${ }^{10}$, S. TASAKA ${ }^{11}$, J. G. Learned ${ }^{12}$, S. Matsuno ${ }^{12}$, S. Smith ${ }^{12}$, K. Martens ${ }^{3}$, M. Vagins ${ }^{3,6}$, Y. Watanabe $^{13}$, T. Hasegawa ${ }^{14}$, T. Ishida ${ }^{14}$, T. Ishi ${ }^{14}$, T. Kobayashi ${ }^{14}$, T. Nakadaira ${ }^{14}$, K. NaKamura ${ }^{3,14}$, K. Nishikawa ${ }^{14}$, H. Nishino $^{14}$, Y. Oyama ${ }^{14}$, K. SaKashita $^{14}$, T. SeKiguchi ${ }^{14}$,

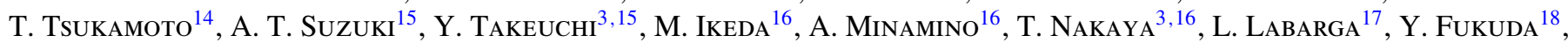

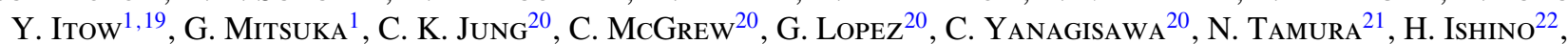

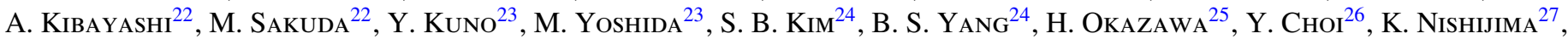

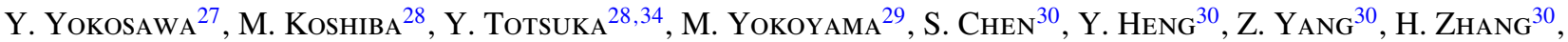

D. Kielczewska ${ }^{31}$, P. Mijakowski ${ }^{31}$, K. Connolly ${ }^{32}$, M. Dziomba ${ }^{32}$, E. Thrane ${ }^{32,35}$, and R. J. Wilkes ${ }^{32}$

(The Super-Kamiokande Collaboration)

${ }^{1}$ Solar-Terrestrial Environment Laboratory, Nagoya University, Nagoya, Aichi 464-8602, Japan

${ }^{2}$ Kamioka Observatory, Institute for Cosmic Ray Research, University of Tokyo, Kamioka, Gifu, 506-1205, Japan

${ }^{3}$ Institute for the Physics and Mathematics of the Universe (IPMU), The University of Tokyo, Kashiwa, Chiba 277-8568, Japan

${ }^{4}$ Research Center for Cosmic Neutrinos, Institute for Cosmic Ray Research, University of Tokyo, Kashiwa, Chiba 277-8582, Japan

${ }^{5}$ Department of Physics, Boston University, Boston, MA 02215, USA

${ }^{6}$ Department of Physics and Astronomy, University of California, Irvine, Irvine, CA 92697-4575, USA

${ }^{7}$ Department of Physics, California State University, Dominguez Hills, Carson, CA 90747, USA

${ }^{8}$ Department of Physics, Chonnam National University, Kwangju 500-757, Korea

${ }^{9}$ Department of Physics, Duke University, Durham, NC 27708, USA

${ }^{10}$ Junior College, Fukuoka Institute of Technology, Fukuoka, Fukuoka 811-0295, Japan

${ }^{11}$ Department of Physics, Gifu University, Gifu, Gifu 501-1193, Japan

12 Department of Physics and Astronomy, University of Hawaii, Honolulu, HI 96822, USA

${ }^{13}$ Physics Division, Department of Engineering, Kanagawa Univers ity, Yokohama, Kanagawa 221-8686, Japan

${ }^{14}$ High Energy Accelerator Research Organization (KEK), Tsukuba, Ibaraki 305-0801, Japan

${ }^{15}$ Department of Physics, Kobe University, Kobe, Hyogo 657-8501, Japan

${ }^{16}$ Department of Physics, Kyoto University, Kyoto 606-8502, Japan

${ }^{17}$ Department of Theoretical Physics, University Autonoma Madrid, Madrid 28049, Spain

${ }^{18}$ Department of Physics, Miyagi University of Education, Sendai, Miyagi 980-0845, Japan

${ }^{19}$ Kobayashi-Maskawa Institute for the Origin of Particle and the Universe, Nagoya University, Nagoya, Aichi 464-8602, Japan

${ }^{20}$ Department of Physics and Astronomy, State University of New York, Stony Brook, NY 11794-3800, USA

${ }^{21}$ Department of Physics, Niigata University, Niigata, Niigata 950-2181, Japan

${ }^{22}$ Department of Physics, Okayama University, Okayama, Okayama 700-8530, Japan

${ }^{23}$ Department of Physics, Osaka University, Toyonaka, Osaka 560-0043, Japan

${ }^{24}$ Department of Physics, Seoul National University, Seoul 151-742, Korea

${ }^{25}$ Department of Informatics in Social Welfare, Shizuoka University of Welfare, Yaizu, Shizuoka, 425-8611, Japan

${ }^{26}$ Department of Physics, Sungkyunkwan University, Suwon 440-746, Korea

${ }^{27}$ Department of Physics, Tokai University, Hiratsuka, Kanagawa 259-1292, Japan

${ }^{28}$ The University of Tokyo, Tokyo 113-0033, Japan

${ }^{29}$ Department of Physics, The University of Tokyo, Tokyo 113-0033, Japan

${ }^{30}$ Department of Engineering Physics, Tsinghua University, Beijing, 100084, China

${ }^{31}$ Institute of Experimental Physics, Warsaw University, 00-681 Warsaw, Poland

${ }^{32}$ Department of Physics, University of Washington, Seattle, WA 98195-1560, USA

Received 2010 December 24; accepted 2011 August 13; published 2011 November 8

\section{ABSTRACT}

We present the result of an indirect search for high energy neutrinos from Weakly Interacting Massive Particle (WIMP) annihilation in the Sun using upward-going muon (upmu) events at Super-Kamiokande. Data sets from SKI-SKIII (3109.6 days) were used for the analysis. We looked for an excess of neutrino signal from the Sun as compared with the expected atmospheric neutrino background in three upmu categories: stopping, non-showering, and showering. No significant excess was observed. The 90\% C.L. upper limits of upmu flux induced by WIMPs of $100 \mathrm{GeV} \mathrm{c}^{-2}$ were $6.4 \times 10^{-15} \mathrm{~cm}^{-2} \mathrm{~s}^{-1}$ and $4.0 \times 10^{-15} \mathrm{~cm}^{-2} \mathrm{~s}^{-1}$ for the soft and hard annihilation channels, respectively. These limits correspond to upper limits of $4.5 \times 10^{-39} \mathrm{~cm}^{-2}$ and $2.7 \times 10^{-40} \mathrm{~cm}^{-2}$ for spin-dependent WIMP-nucleon scattering cross sections in the soft and hard annihilation channels, respectively.

Key words: dark matter - neutrinos

Online-only material: color figures 


\section{INTRODUCTION}

From recent observations, Weakly Interacting Massive Particles (WIMPs) are considered to be a favorite candidate for cold dark matter (Bennett et al. 2003). From the viewpoint of minimum extensions of the Standard Model to a supersymmetric model (SUSY), the most well-motivated candidate for WIMPs in the universe is the lightest supersymmetric neutral particle (LSP; Jungman et al. 1996; Bergström 2000).

One candidate for the LSP which is theoretically well studied is the neutralino ( $\widetilde{\chi})$ (Drees \& Nojiri 1993). The neutralino is a linear combination of supersymmetric particles that mix after electroweak-symmetry breaking. Taking into account constraints from accelerator experiments and astronomical observations, the neutralino mass is expected to be in the range from several tens of $\mathrm{GeV}$ to $10 \mathrm{TeV}$, depending on the model assumed for SUSY breaking and on the SUSY parameters (Amsler et al. 2008; Gilmore 2007).

One method of searching for a WIMP dark matter signal is an indirect search where decay or annihilation products from WIMPs are observed as originating from the center of a gravitational potential well such as a celestial body. WIMPs have a small but finite probability of elastic scattering with a nucleus. As matter is concentrated in deep gravitational potential wells, this scattering is more likely to occur near the centers of such wells; if their final velocities after the scattering are less than the escape velocity, they are trapped gravitationally and eventually accumulated in the center of the gravitational potential. There are two types of scattering: spin-dependent (SD) and spin-independent (SI) scattering. In the former, WIMPs couple to the spin of the target nucleus, and in the latter WIMPs coherently scatter on the nucleus with probability scaling as the square of the mass of the target nucleus. WIMPs, being Majorana particles, can annihilate in pairs and produce primarily $\tau$ leptons; $b, c$, and $t$ quarks; gauge bosons; and Higgs bosons depending upon their masses and compositions. At the final step, the decay of the WIMP annihilation products may produce many kinds of particles such as neutrinos, positrons, antiprotons, antideuterons, etc.

In the Sun, WIMPs are most likely to scatter on the dominant component, hydrogen, either via SD or SI coupling. Direct detection searches on Earth turn out to have a sensitivity advantage, generally, for SI interactions, while indirect searches apply to both (Kamionkowski 1995). WIMP annihilation products in the form of muon neutrinos are an excellent instrument for indirect searches since they can pass through the matter of the Sun, and interact in the Earth; the resulting detected muon will be correlated with its parent neutrino direction, providing an unmistakable signal. In the past, several neutrino telescopes such as Super-Kamiokande, AMANDA, and IceCube have reported the results of indirect searches for WIMPs in the Sun (Desai et al. 2004; Braun \& Hubert 2009; Abbasi et al. 2009). Here we investigate upward-going muons (upmus) which are generated from high energy neutrinos come from the WIMP annihilations using the Super-Kamiokande (Super-K, SK). We search in the direction of the Sun, and an excess of neutrino flux above the atmospheric neutrino background is sought in the upmu events. Although recent new detectors such as IceCube have larger acceptances for higher mass WIMPs, SK is better equipped for

\footnotetext{
${ }^{33}$ Deceased.

${ }^{34}$ Deceased.

${ }^{35}$ Present address: Department of Physics and Astronomy, University of Minnesota, MN, 55455, USA.
}

the search of lower mass WIMPs $(<100 \mathrm{GeV})$ due to its lower energy threshold for neutrino signals.

A previous WIMP search in SK was done in 2004 using a data set of 1679.6 days of through-going upmu events (Desai et al. 2004). In this paper, we have nearly doubled the size of the data set (3109.6 days). In addition, we have divided the upmu events into three categories, taking into account their neutrino energy dependence of detector response in order to improve the limit on the WIMP-induced upmu flux and SD cross section, especially for lower mass WIMPs. This analysis now also uses WIMPsim (Blennow et al. 2008) and the DARKSUSY (Gondolo et al. 2004) simulator in the calculation in order to simulate the WIMP-induced neutrino fluxes and propagation.

\section{INDIRECT WIMP SEARCH IN SUPER-KAMIOKANDE}

The SK detector is a 50 kton water Cherenkov detector located at the Kamioka mine in Japan. Since SK started operation in 1996, there have been four experimental phases: SK-I (1996 April-2001 July), SK-II (2002-2005 October), SK-III (2006 July-2008 August), and SK-IV(2008 September-). We use the data sets from SK-I to SK-III (3109.6 days) for this analysis.

Upmu events, which are categorized as the most energetic events in SK, are used for the analysis. Muon neutrinos interact in the rock surrounding the detector to produce muons via charged current interactions. If the energy of the incoming neutrino is high enough, the muon produced by the neutrino will enter the detector and emit Cherenkov light. To separate neutrino-induced muons from cosmic ray muons, we select only upmus, since the background from downward-going cosmic ray muons overwhelms any neutrino-induced muons from above. Both the neutrino cross section and the range of the produced muon are proportional to the neutrino energy. Thus, the effective target volume is very much increased if we use the upmu events for high energy neutrino searches. Upmu events are divided into three categories: stopping, non-showering through-going, and showering through-going. If upmus stop in the detector, they are categorized as "stopping." Stopping upmus are the lowest energy portion of the upmu sample. Upmus that fully traverse the detector are classified as "through-going." The category of through-going is then further subdivided into "showering" (if the event has accompanying radiation due to radiative energy loss) and "non-showering" (otherwise). The details of the showering versus non-showering classification scheme are described in Desai et al. (2008). Showering through-going events make up the highest energy events in the upmu sample. The detector angular resolution is $1^{\circ}$ for stopping and non-showering through-going, and 1.4 for showering through-going muons, as estimated by Monte Carlo (MC) simulation. This analysis is based on a total of 4351 upmus: 919 stopping, 2901 non-showering throughgoing, and 531 showering through-going.

The main background source for this analysis is upmu events produced by atmospheric neutrinos. These were simulated by the NEUT neutrino simulation package (Hayato 2009) and a GEANT-based detector simulator. We use the neutrino flux calculated by Honda et al. $2007\left(E_{v}<10 \mathrm{TeV}\right)$ and Volkova $1980\left(E_{v}>10 \mathrm{TeV}\right)$ for this simulation. The main uncertainty in the absolute number of upmus comes from the uncertainty in the cosmic ray flux $(\approx 20 \%)$. In this analysis, this uncertainty is irrelevant since we normalize the absolute number of simulated atmospheric neutrino events by the number of upmu data events in each category. The effect of neutrino oscillation is also taken into account in the simulation. We consider $v_{\mu}-v_{\tau}$ oscillations 

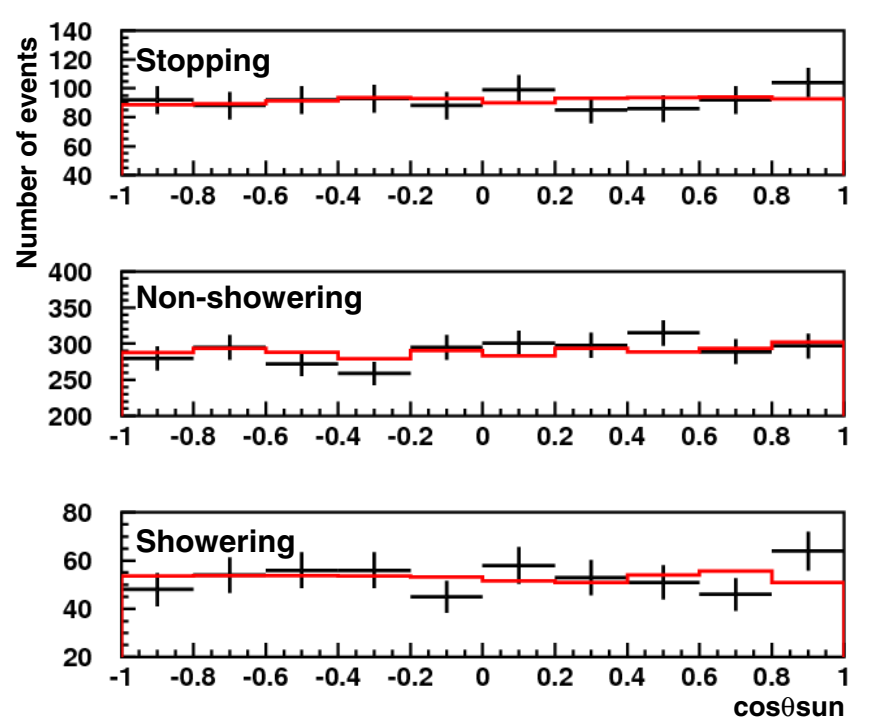

Figure 1. Distribution for $\cos \theta_{\text {sun }}$ of upmu events relative to the Sun. Here, $\cos \theta_{\text {sun }}=1$ is the direction of the Sun. Crosses indicate the data from SKI-III (3109.6 days) and the histogram indicates atmospheric neutrino MC with $v_{\mu}-v_{\tau}$ oscillations normalized by the total number of data events in each category.

(A color version of this figure is available in the online journal.)

with $\Delta m^{2}=0.0025 \mathrm{eV}^{2}$ and $\sin ^{2} \theta=1.0$ independently obtained from the previous analyses (Ashie et al. 2005, 2004).

\section{RESULTS OF THE WIMP SEARCH}

\subsection{Uрти Events from the Sun}

The event distribution (SKI-III:3109.6 days) as a function of $\cos \theta_{\text {sun }}$ for each category of upmu is shown in Figure 1. Here, $\theta$ is the angle between the reconstructed upmu direction and the direction of the Sun. This $\theta$ is called the "cone halfangle." In Figure $1, \cos \theta_{\text {sun }}=1$ is defined as the direction of the Sun. To compare data with the MC events, each MC event was assigned a time sampled from the observed upmu arrival time in order to match the live-time distribution and the detector acceptance of the observed events. The number of MC events is normalized to the total number of data events for each category. Here the normalization was done for the entire $\cos \theta_{\text {sun }}$ region for simplicity. Without signal regions the difference of normalization is less than $1 \%$.

We search for an excess from the direction of the Sun in each of the three upmu categories: stopping (A), nonshowering (B), and showering (C). The fraction of each upmu category as a function of parent neutrino energy $\left(E_{v}\right)$ is shown in Figure 2. For typical energies of 10, 100, and $1000 \mathrm{GeV}$, they are $\mathrm{A}: \mathrm{B}: \mathrm{C}=0.73: 0.20: 0.07,0.10: 0.62: 0.28$, and 0.02:0.50:0.48, respectively. These fractions were obtained from the atmospheric neutrino MC simulation. The fraction depends only on parent neutrino energy; its source is irrelevant. We can roughly deduce the WIMP mass from the event category where an excess is observed. Since the spectrum of the atmospheric neutrino background is proportional to $E^{-2.7}$, while the signal spectrum from WIMPs gives a bump structure, this energydependent approach is good for reducing the atmospheric neutrino background. We separately analyze the three upmu samples, then combine the results by taking into account each fraction assuming a WIMP mass.

Since the directional correlation between neutrinos and their produced leptons depends on neutrino energy, it also depends

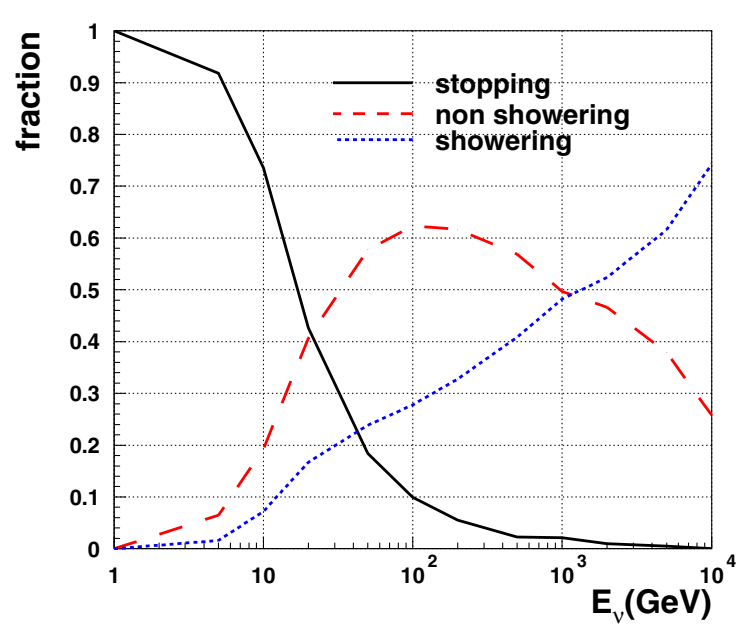

Figure 2. Fraction of each upmu category as a function of the parent neutrino energy $\left(E_{v}\right)$. Three upmu categories are shown as follows: stopping (black solid line), non-showering (red dashed line), and showering (blue dotted line). The total of the fractions for the three categories is normalized to 1. A Monte Carlo simulation for atmospheric neutrinos is used to calculate this.

(A color version of this figure is available in the online journal.)
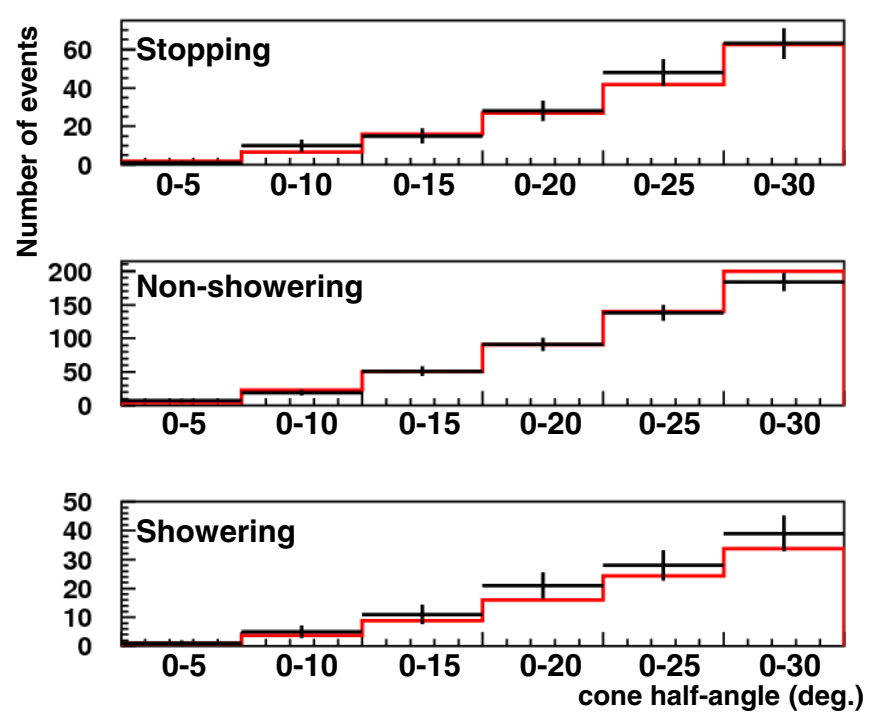

Figure 3. Number of events observed in each cone half-angle toward the Sun. Here, zero degrees corresponds to the direction of the Sun. Crosses indicate the data from SKI-III (3109.6 days) and the histogram indicates atmospheric neutrino MC with $v_{\mu}-v_{\tau}$ oscillations normalized to the total number of data events in each category.

(A color version of this figure is available in the online journal.)

on the WIMP mass and its decay mode. Therefore, we look for a possible correlation of upmu direction to the Sun by searching for an excess of events in various cone half-angles up to $30^{\circ}$. Using DARKSUSY, we checked the neutrino-muon angular correlation for the neutrino spectrum generated by WIMP annihilation in the Sun for various SUSY parameters and found that the angle between the two is smaller than $30^{\circ}$ for most cases. The number of events observed in each cone half-angle toward the Sun is shown in Figure 3. Zero degree corresponds to the direction of the Sun. Crosses indicate the data from SKI-III (3109.6 days) and the histogram indicates atmospheric neutrino MC with same normalization as in Figure 1. 
Table 1

Event Summary to Calculate the Flux Limit $(\phi)$ Assuming the Soft Annihilation Channel is Dominant

\begin{tabular}{|c|c|c|c|c|c|c|c|c|c|c|c|c|}
\hline \multirow[b]{2}{*}{$\begin{array}{l}m_{\chi} \\
(\mathrm{GeV})\end{array}$} & \multirow[b]{2}{*}{$\begin{array}{c}\theta \\
(\operatorname{deg})\end{array}$} & \multicolumn{3}{|c|}{ Stopping } & \multicolumn{3}{|c|}{ Non-showering } & \multicolumn{3}{|c|}{ Showering } & \multirow[b]{2}{*}{$N_{90}$} & \multirow[b]{2}{*}{$\begin{array}{l}\phi\left(\times 10^{-15}\right) \\
\left(\mathrm{cm}^{-2} \mathrm{~s}^{-1}\right)\end{array}$} \\
\hline & & $n_{\mathrm{obs}}$ & $n_{\mathrm{BG}}$ & $\begin{array}{c}F \\
(\%)\end{array}$ & $n_{\mathrm{obs}}$ & $n_{\mathrm{BG}}$ & $\begin{array}{c}F \\
(\%)\end{array}$ & $n_{\mathrm{obs}}$ & $n_{\mathrm{BG}}$ & $\begin{array}{c}F \\
(\%)\end{array}$ & & \\
\hline 10 & 30 & 63 & 62.5 & 98 & 184 & 200.3 & 2 & 39 & 33.7 & 0 & 14.8 & 8.9 \\
\hline 100 & 10 & 10 & 6.7 & 78 & 19 & 22.5 & 16 & 5 & 3.8 & 6 & 10.9 & 6.5 \\
\hline 1000 & 6 & 3 & 2.7 & 59 & 8 & 7.2 & 29 & 1 & 1.4 & 12 & 6.41 & 3.8 \\
\hline 10000 & 5 & 1 & 1.9 & 28 & 7 & 4.6 & 52 & 1 & 0.9 & 20 & 6.99 & 4.2 \\
\hline
\end{tabular}

Notes. The numbers of events $\left(n_{\mathrm{obs}}\right)$ and estimated background $\left(n_{\mathrm{BG}}\right)$ for each cone half-angle $(\theta)$ which corresponds to a certain WIMP mass $\left(m_{\chi}\right)$ are shown for all categories of upmu. This cone half-angle is defined by the criterion that more than $90 \%$ of the WIMP-induced upmus are contained for the assumed WIMP masses. We also show the estimated fraction $(F)$ of each upmu category for the given WIMP mass. The calculated result of $N_{90}$ and the flux limit for each WIMP mass are shown in the last two columns.

Table 2

Event Summary and Upmu Flux Limit Assuming the Hard Annihilation Channel is Dominant

\begin{tabular}{lcccccccccccc}
\hline \hline & & \multicolumn{3}{c}{ Stopping } & \multicolumn{3}{c}{ Non-showering } & \multicolumn{3}{c}{ Showering } \\
\hline $\begin{array}{l}m_{\chi} \\
(\mathrm{GeV})\end{array}$ & $\begin{array}{c}\theta \\
(\mathrm{deg})\end{array}$ & $n_{\mathrm{obs}}$ & $n_{\mathrm{BG}}$ & $\begin{array}{c}F \\
(\%)\end{array}$ & $n_{\mathrm{obs}}$ & $n_{\mathrm{BG}}$ & $\begin{array}{c}F \\
(\%)\end{array}$ & $n_{\mathrm{obs}}$ & $n_{\mathrm{BG}}$ & $\begin{array}{c}F \\
(\%)\end{array}$ & $\begin{array}{c}N_{90} \\
\phi\left(\times 10^{-15}\right) \\
\left(\mathrm{cm}^{-2} \mathrm{~s}^{-1}\right)\end{array}$ \\
\hline 80.3 & 8 & 4 & 4.2 & 44 & 14 & 13.4 & 40 & 3 & 2.5 & 16 & 8.4 & 5.0 \\
100 & 7 & 3 & 3.3 & 42 & 10 & 9.9 & 41 & 2 & 2.2 & 17 & 6.9 & 4.1 \\
1000 & 3 & 0 & 0.5 & 40 & 2 & 2.1 & 41 & 1 & 0.3 & 19 & 4.2 & 2.5 \\
10000 & 3 & 0 & 0.5 & 24 & 2 & 2.1 & 54 & 1 & 0.3 & 22 & 4.5 & 2.7 \\
\hline
\end{tabular}

Note. The meaning of each column is the same as in Table 1.

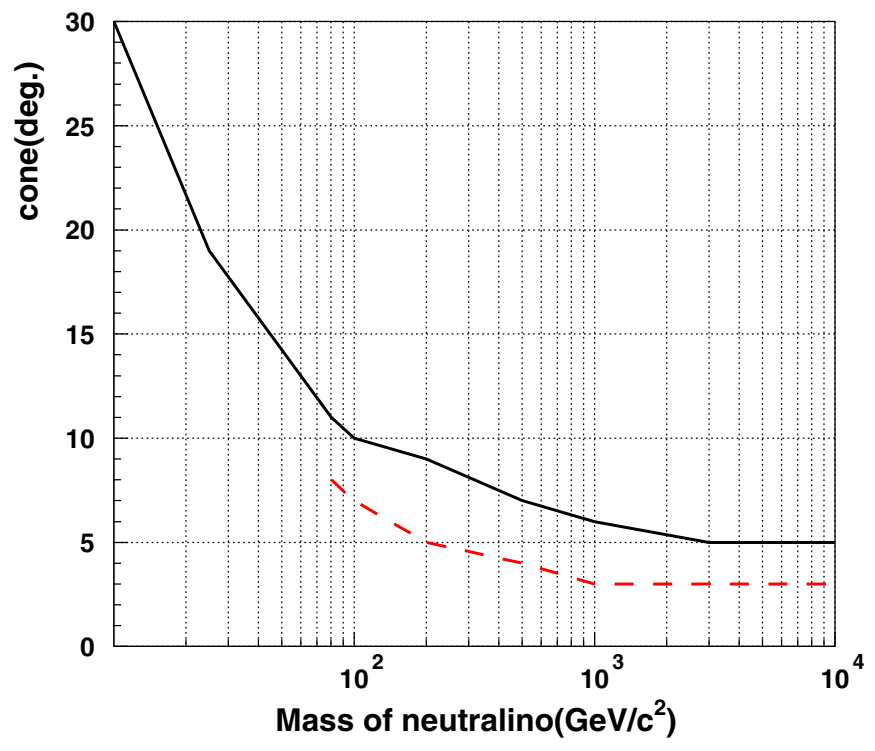

Figure 4. Cone half-angle which contains more than $90 \%$ of the total number of upmus expected from WIMP annihilations in the Sun when we assume the soft annihilation channel (black solid line) and hard annihilation channel (red dashed line). This result was obtained from WIMPsim.

(A color version of this figure is available in the online journal.)

\subsection{Uрти Flux Limit from the Sun}

We calculate the upper limit of upmu flux from the direction of the Sun with respect to the WIMP mass. Here we estimate the cone half-angles which contain more than $90 \%$ of the total number of upmus expected from WIMP annihilations in the Sun. We use the WIMPsim package (Blennow et al. 2008), which simulates WIMP annihilations, propagation of neutrinos from those annihilations, and the propagation of muons after neutrino interactions in the rock. In order to derive the expected neutrino spectrum from the WIMP annihilation, we consider two cases: annihilation into $b \bar{b}$ (soft channel) and annihilation into $W^{+} W^{-}$(hard channel). These give the softest and hardest neutrino spectra, respectively. The cone half-angles obtained from these spectra are shown in Figure 4. The cone half-angle for the soft channel is generally wider at the same WIMP mass since many neutrinos from annihilation via the soft channel are expected to have lower energy.

The other smearing effects for angular correlation are muon propagation in the surrounding rocks, distribution of WIMPs in the Sun, and angular resolution of upmu reconstruction. The smearing due to Coulomb multiple scattering in the surrounding rocks is $2^{\circ}-3^{\circ}$ for muons with energy above $1 \mathrm{GeV}$. The angular size of the Sun at the Earth is 0.5, which is negligible (Edsjö 1997). These two smearing effects were taken into account by WIMPsim. The detector angular resolution of $1^{\circ}$ (stopping and non-showering) and 1.4 (showering) are smaller than the smallest cone half-angle and thus were not taken into account.

We show the cone half-angle for several WIMP masses in Tables 1 and 2. Table 2 begins at $80.3 \mathrm{GeV}$ since WIMPs with mass lower than $80.3 \mathrm{GeV}$ cannot annihilate into $W^{+} W^{-}$. We followed the DarkSUSY for the treatment of masses of standard model particles (Blennow et al. 2008).

For each cone half-angle, which corresponds to a certain WIMP mass in each annihilation channel, we calculated the upper Poissonian limit of the upmu flux:

$\phi(90 \%$ C.L. $)=\frac{N_{90}\left(n_{\mathrm{obs}}^{1}, n_{\mathrm{BG}}^{1}, F^{1}, n_{\mathrm{obs}}^{2}, n_{\mathrm{BG}}^{2}, F^{2}, n_{\mathrm{obs}}^{3}, n_{\mathrm{BG}}^{3}, F^{3}\right)}{\epsilon \times A \times T}$,

where $A$ is the detector cross-sectional area in the direction of the expected signal averaged over Sun directions. For each position of the Sun, this area is calculated using a minimal track length of $7 \mathrm{~m}$ in the inner detector as a criterion to select viable upmu trajectories. It varies from $968 \mathrm{~m}^{2}$ to $1290 \mathrm{~m}^{2}$ with the 

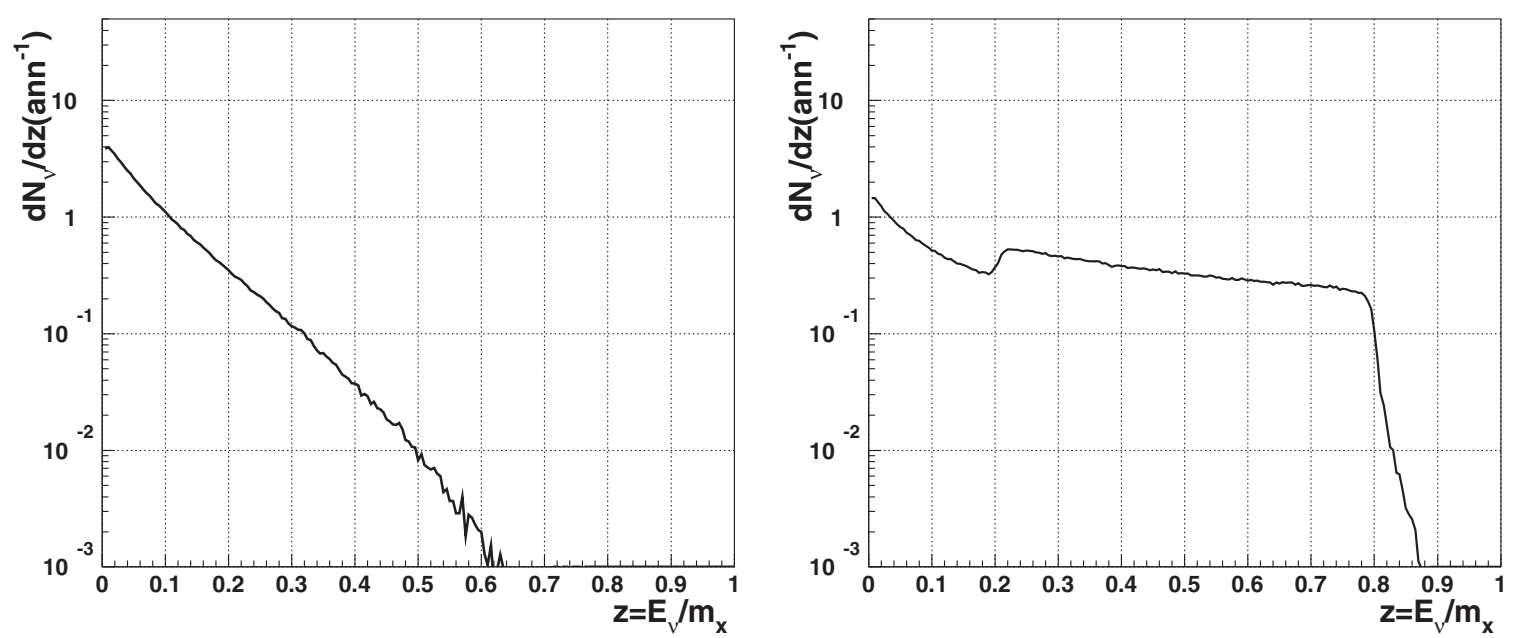

Figure 5. Simulated neutrino flux $\left(v_{\mu}+\overline{v_{\mu}}\right)$ at the surface of the detector from the annihilation of neutralinos of $100 \mathrm{GeV}$ in the soft annihilation channel alone (left panel) and hard annihilation channel alone (right panel).

position of the Sun. $T$ is the experimental live time and $\epsilon$ is the detection efficiency. For upmu samples, the inefficiency of detection is negligible (Desai et al. 2008). The average exposure $A \times T$ is $1.67 \times 10^{11} \mathrm{~m}^{2} \mathrm{~s} . N_{90}$ is the upper Poissonian limit on the number of upmu events at $90 \%$ C.L., given the number of observed events of each category $\left(n_{\mathrm{obs}}^{i}\right)$, expected background of each category $\left(n_{\mathrm{BG}}^{i}\right)$ and fraction of each category $\left(F^{i}\right)$, where the index $i$ corresponds to the three types of upmu category. This fraction is estimated from the neutrino spectrum of the given WIMP mass for each annihilation channel and the fraction of the upmu categories as a function of the parent neutrino energy as shown in Figure 2. The neutrino spectra were simulated by WIMPsim. Figure 5 shows the simulated neutrino spectra for the soft and hard annihilation channels, assuming a neutralino mass of $100 \mathrm{GeV}$.

$N_{90}$ was calculated combining all categories of upmu for a certain mass based on Bayes' theorem (Amsler et al. 2008). The Poisson-based likelihood function for the expected signal is as follows:

$$
L\left(n_{\mathrm{obs}}^{i} \mid v_{s}\right)=\prod_{i=0}^{3} \frac{\left(v_{s} F^{i}+n_{\mathrm{BG}}^{i}\right)^{n_{\mathrm{obs}}^{i}}}{n_{\mathrm{obs}}^{i} !} e^{-\left(v_{s} F^{i}+n_{\mathrm{BG}}^{i}\right)},
$$

where $v_{s}$ is the expected real signal. Using the likelihood function in Equation (2), the upper confidence level is written as

$$
\text { C.L. }=\frac{\int_{v_{s}=0}^{N_{\text {limit }}} L\left(n_{\mathrm{obs}}^{i} \mid v_{s}\right) d v_{s}}{\int_{v_{s}=0}^{\infty} L\left(n_{\mathrm{obs}}^{i} \mid v_{s}\right) d v_{s}} .
$$

Applying $90 \%$ as the C.L. in Equation (3), the upper Poissonian limit $\left(N_{90}\right)$ is obtained.

The summaries of the numbers of upmu events and the estimated backgrounds for the cone half-angles that correspond to each WIMP mass, $N_{90}$, and the calculated flux limits are shown in Tables 1 and 2.

The $90 \%$ confidence level flux limits as a function of WIMP mass are shown in Figures 6 and 7. Results from other experiments, AMANDA (Braun \& Hubert 2009) and IceCube (Abbasi et al. 2009), are also shown in these figures. The previous Super-K result (Desai et al. 2004) is also shown in Figure 6, but please note that it was calculated assuming an annihilation branching ratio. That result assumed that $80 \%$ of the annihilation products were $b \bar{b}, 10 \%$ were $c \bar{c}$, and $10 \%$ are

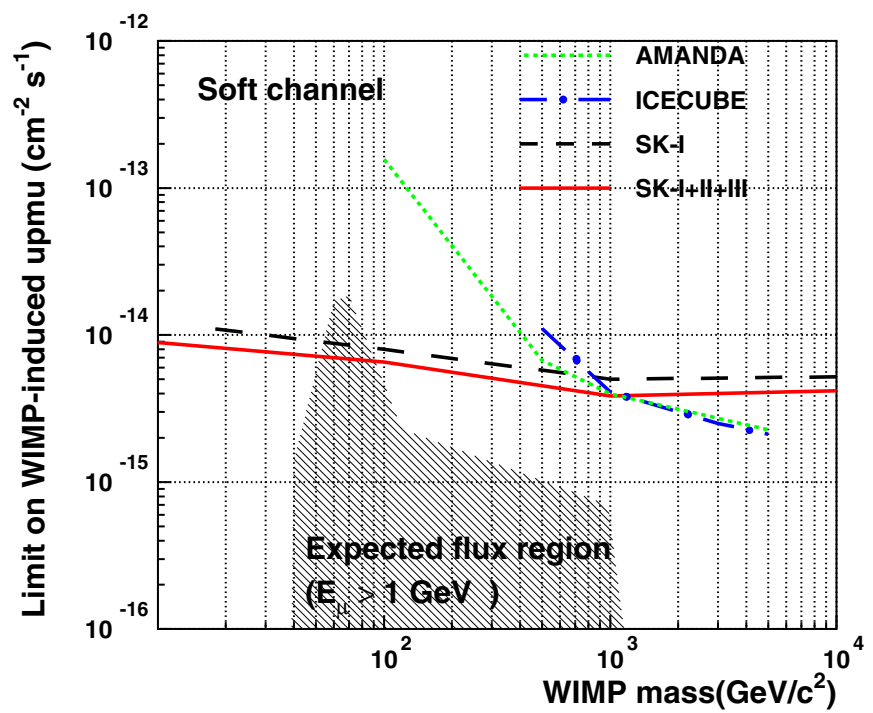

Figure 6. Upmu flux limit vs. WIMP mass for the soft annihilation channel. The flux limits of AMANDA (Braun \& Hubert 2009; green dotted line), IceCube (Abbasi et al. 2009; blue dot-dash line), and this analysis (red solid line) are shown. Also shown is the previous limit from Super-K (Desai et al. 2004; black dashed line). Note that SK's previous limit does not assume the pure soft channel, but assumes a typical annihilation channel in which the branching ratio for the soft annihilation channel is relatively high (80\%). The shaded region is the expected flux region from DARKSUSY.

(A color version of this figure is available in the online journal.)

$\tau \bar{\tau}$, as a typical branching ratio. We cannot regard this as a pure soft annihilation, but it is shown for reference.

\subsection{Limit on WIMP-proton SD Cross Section}

From the upmu flux limits shown in Figures 6 and 7, we also calculate the upper limit on the SD scattering cross section of WIMPs. If we assume that the capture rate of WIMPs and the WIMP annihilation rate are in equilibrium in the Sun, the neutrino flux from annihilation depends only on the capture rate. Since the age of the Sun is much longer than the timescale of the annihilation, it is a safe assumption that they are in equilibrium. We can relate the upmu flux, $\Phi_{\mu}^{f}$, to the SD cross section, $\sigma^{\mathrm{SD}}$, on the assumption that only one annihilation channel is dominant and the SI cross section is zero. The $\mathrm{SD}$ cross section, $\sigma^{\mathrm{SD}}$, can 


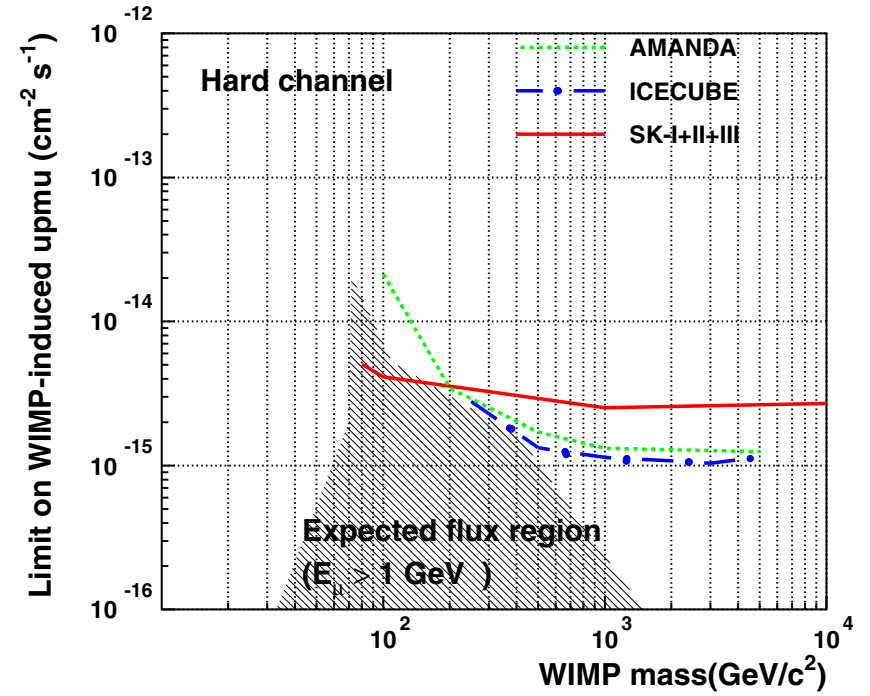

Figure 7. Upmu flux limit vs. WIMP mass for the hard annihilation channel. The flux limits of AMANDA (Braun \& Hubert 2009; green dotted line), IceCube (Abbasi et al. 2009; blue dot-dash line), and this analysis (red solid line) are shown. The shaded region is the expected flux region from DARKSUSY.

(A color version of this figure is available in the online journal.)

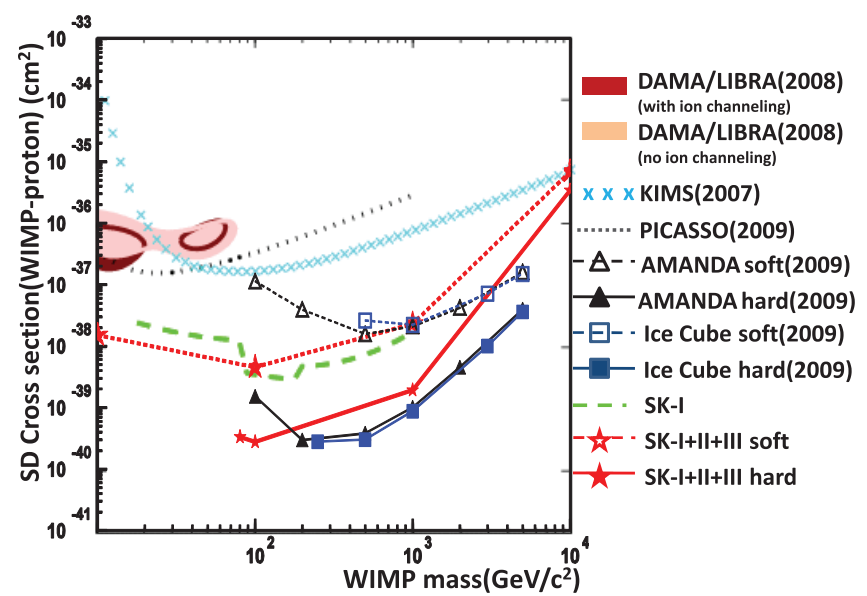

Figure 8. Limit on the WIMP-proton spin-dependent cross section as a function of WIMP mass. Limits from direct detection experiments: DAMA/LIBRA allowed region (Savage et al. 2009; dark red and light red filled, for with and without ion channeling, respectively), KIMS (Lee et al. 2007; light blue crosses), and PICASSO (Archambault et al. 2009; gray dotted line) are shown. Also we show here the results of indirect detection (neutrino telescopes): AMANDA (Braun \& Hubert 2009; black line with triangles), IceCube (Wikström \& Edsjö 2009; blue line with squares), and this analysis (red line with stars). The previous limit from Super-K (green dashed line) is also shown. This plot was made from the SUSY Dark Matter/Interactive Direct Detection Plotter, http://dmtools.berkeley.edu/limitplots.

(A color version of this figure is available in the online journal.)

be written as (Wikström \& Edsjö 2009)

$$
\sigma^{\mathrm{SD}}=\kappa_{f}^{\mathrm{SD}}\left(m_{\chi}\right) \Phi_{\mu}^{f}
$$

where $\kappa_{f}^{\mathrm{SD}}\left(m_{\chi}\right)$ is the conversion factor between the SD cross section and the muon flux. This value depends on the WIMP mass, $m_{\chi}$. We use the factor introduced in Figure 3 of Wikström $\&$ Edsjö (2009), which was calculated using DARKSUSY.

The calculated upper limit is shown in Figure 8. Also shown are results from direct detection experiments: DAMA/LIBRA allowed region (Savage et al. 2009), KIMS (Lee et al. 2007), and PICASSO (Archambault et al. 2009); and from neutrino telescopes: AMANDA (Braun \& Hubert 2009) and IceCube (Abbasi et al. 2009). All of the limits from the neutrino telescopes (including our limit) are calculated using the same method described in Wikström \& Edsjö (2009). The previous limit from Super-K is also shown for reference. Note that the gaps at WIMP masses of $\sim 80 \mathrm{GeV}$ and $174 \mathrm{GeV}$ in this previous result are due to the thresholds of the $W^{+} W^{-}$and $\tau \bar{\tau}$ annihilation channels. These do not appear in the new result because we assume only one annihilation channel. Our limit is improved at low WIMP mass (below $100 \mathrm{GeV}$ ), which is stronger than limits from other indirect-detection experiments. Our limit is also stronger than direct-detection experiments.

\section{CONCLUSION}

We have performed an updated indirect search for WIMPs using the Super-Kamiokande data of SKI-III (3109.6 days). A WIMP-induced neutrino signal was searched for in the direction of the Sun.

We compared upmu events with the estimated background of atmospheric neutrinos. No significant excess was observed.

To calculate the upmu flux limit, we used a new method involving WIMPsim and the DARKSUSY simulator. Cone halfangles that contained more than $90 \%$ upmu signal for each WIMP mass were extracted from simulation. Upper upmu flux limits as a function of WIMP mass for the soft and the hard annihilation channels were obtained. Our limit extends into the region of expected upmu flux estimated by some SUSY models.

An upper limit on the SD scattering cross section was also obtained. We used a WIMP mass-dependent conversion factor as described in Wikström \& Edsjö (2009). At low WIMP mass (below $100 \mathrm{GeV}$ ), our limit on the WIMP-proton SD cross section is better than other experiments for both direct-detection and indirect-detection experiments.

\section{REFERENCES}

Abbasi, R., Abdou, Y., Ackermann, M., et al. 2009, Phys. Rev. Lett., 102, 201302 Amsler, C., Doser, M., Antonelli, M., et al. 2008, Phys. Lett. B, 667, 1

Archambault, S., Aubin, F., Auger, M., et al. 2009, Phys. Lett. B, 682, 185 Ashie, Y., Hosaka, J., Ishihara, K., et al. 2004, Phys. Rev. Lett., 93, 101801 Ashie, Y., Hosaka, J., Ishihara, K., et al. 2005, Phys. Rev. D, 71, 112005 Bennett, C. L., Halpern, M., Hinshaw, G., et al. 2003, ApJS, 148, 1 Bergström, L. 2000, Rep. Prog. Phys., 63, 793

Blennow, M., Edsjö, J., \& Ohlsson, T. 2008, J. Cosmology Astropart. Phys., JCAP01(2008)021

Braun, J., \& Hubert, D. for the Ice Cube Collaboration. 2009, arXiv:0906.1615 Desai, S., Ashie, Y., Fukuda, S., et al. 2004, Phys. Rev. D, 70, 083523

Desai, S., Abe, K., Hayato, Y., et al. 2008, Astropart. Phys., 29, 42

Drees, M., \& Nojiri, M. M. 1993, Phys. Rev. D, 47, 376

Edsjö, J. 1997, PhD thesis, Uppsada University (arXiv:astro-ph/9704384)

Gilmore, R. C. 2007, Phys. Rev. D, 76, 043520

Gondolo, P., Edsjö, J., Ullio, P., et al. 2004, J. Cosmol. Astropart. Phys., JCAP07(2004)008

Hayato, Y. 2009, Acta Phys. Polon, B40, 2477

Honda, M., Kajita, T., Kasahara, K., Midorikawa, S., \& Sanuki, T. 2007, Phys. Rev. D, 75, 043006

Jungman, G., Kamionkowski, M., \& Griest, K. 1996, Phys. Rep., 267, 195

Kamionkowski, M. 1995, Phys. Rev. Lett., 74, 5174

Lee, H. S., Bhang, H. C., Choi, J. H., et al. 2007, Phys. Rev. Lett., 99, 091301

Savage, C., Gelmini, G., Gondolo, P., \& Freese, K. 2009, J. Cosmol. Astropart. Phys., JCAP04(2009)010

Volkova, L. V. 1980, Sov. J. Nucl. Phys., 31, 784

Wikström, G., \& Edsjö, J. 2009, J. Cosmol. Astropart. Phys., JCAP04(2009)009 